\title{
VOLUMES OF VECTOR FIELDS ON SPHERES
}

\author{
SHARON L. PEDERSEN
}

\begin{abstract}
In this paper we study the problem: What is the unit vector field of smallest volume on an odd-dimensional sphere? We exhibit on each sphere a unit vector field with singularity which has exceptionally small volume on spheres of dimension greater than four. We conjecture that this volume is the infimum for volumes of bona fide unit vector fields, and is only achieved by the singular vector field.

We generalize the construction of the singular vector field to give a family of cycles in Stiefel manifolds, each of which is a smooth manifold except for one singular point. Except for some low-dimensional cases, the tangent cones at these singular points are volume-minimizing; and half of the cones are nonorientable. Thus, we obtain a new family of nonorientable volume-minimizing cones.
\end{abstract}

\section{INTRODUCTION}

What are the optimal unit vector fields on a round unit sphere of odd dimension?

Here, optimal is taken to mean of minimum volume as a submanifold of the unit tangent bundle. On a flat torus, the optimal unit vector fields are the parallel ones. On the round unit $(2 k+1)$-sphere $S^{2 k+1}$, no parallel vector fields exist, but we can seek the best organized vector fields. Their organization is rewarded by small volume.

Initial results on this problem are due to Gluck and Ziller, who showed [3] that the Hopf vector fields are the unique optimal unit vector fields on $S^{3}$; and to Johnson, who showed [4] that on $S^{5}$ the Hopf vector fields are only unstable critical points of volume, and thus no longer optimal.

In this paper, we show that, beginning on $S^{5}$, there exist unit vector fields of exceptionally small volume, converging to a vector field with one singularity. This result suggests the possibility that there exist no unit vector fields of minimum volume on $S^{n}$ for $n \geq 5$. We conjecture that this is so, and, moreover, that the limiting vector field with singularity is of minimum volume in its homology class in the unit tangent bundle.

The construction of a singular vector field on $S^{n}$ can be generalized to give a singular field of orthonormal $(k-1)$-frames on $S^{n}$. Such a field can be viewed as a certain cycle in the Stiefel manifold $V_{k} \mathbf{R}^{n+1}$ of orthonormal $k$-frames in $\mathbf{R}^{n+1}$. In general, this cycle has one nonmanifold point. An infinite family of

Received by the editors May 3, 1990 and, in revised form, October 18, 1990.

1980 Mathematics Subject Classification (1985 Revision). Primary 53C20, 53C65; Secondary $58 \mathrm{E} 15$. 
new examples of volume-minimizing cones is provided by the tangent cones at the singular points of these cycles. This family of cones includes an infinite family of unorientable cones.

\section{HISTORY AND DEFINITIONS}

The volume of a vector field $V$ on a Riemannian manifold $M$ is defined to be its volume measured as a submanifold of the tangent bundle $T M$, using the metric induced on $T M$ by the metric on $M$. Letting $d \mu$ be the volume form on $M$, we have

$$
\text { Vol } V=\int_{M} \sqrt{\operatorname{det}\left(I+(\nabla V)(\nabla V)^{t}\right)} d \mu .
$$

Thus, Vol $V \geq \operatorname{Vol} M$, with equality if and only if $V$ is parallel. We define an optimal vector field on $M$ to be a unit vector field of minimum volume among all unit vector fields on $M$. (The methods used in this paper tend to look for minima within a given homology class; however, since all unit vector fields on a sphere lie in the same homology class, this will not be an obstacle here.) An optimal vector field on a manifold need not exist, nor, if one does exist, need it be unique.

Define a Hopf vector field, $H^{2 k+1}$, to be a unit vector field tangent to the fibers of a Hopf fibration of $S^{2 k+1}$. Many beautiful properties of the Hopf fibrations are described in [2]. The volumes of the Hopf vector fields are computed in [3] to be $\mathrm{Vol} H^{2 k+1}=2^{k} \mathrm{Vol} S^{2 k+1}$, and the following theorem is proved.

Theorem 1 (Gluck-Ziller). The optimal unit vector fields on $S^{3}$ are precisely the Hopf vector fields.

However, it is shown in [4] that

Theorem 2 (Johnson). The Hopf vector fields on $S^{2 k+1}$ are unstable for $k>1$.

A (nonsharp) lower bound for the volume of any unit vector field $V$ on $S^{2 k+1}$ was given in [3]:

$$
\operatorname{Vol} V>c(k)=\sum_{i=0}^{k}\left(\begin{array}{c}
k \\
i
\end{array}\right)^{2} /\left(\begin{array}{c}
2 k \\
2 i
\end{array}\right) .
$$

We simplify the constant $c(k)$ as follows. Let $a_{i}=\left(\begin{array}{c}2 i \\ i\end{array}\right)$. Rewrite $c(k)$ as $c(k)=\left(\sum_{k} a_{i} a_{k-i}\right) /\left(\begin{array}{c}2 k \\ k\end{array}\right)$. Notice that $f(x)=1 / \sqrt{1-4 x}=\sum_{k} a_{k} x^{k}$, and so $(f(x))^{2}=\sum_{i, k} a_{i} a_{k-i} x^{k}$. But also $(f(x))^{2}=1 /(1-4 x)=\sum_{k} 4^{k} x^{k}$; hence we obtain $c(k)$ in closed form: $c(k)=4 k /\left(\begin{array}{c}2 k \\ k\end{array}\right)$. Applying Stirling's formula, $n ! \sim(n / e)^{n} \sqrt{2 \pi n}$, shows that $c(k) \sim \sqrt{\pi k}$.

\section{A SINGULAR VECTOR FIELD}

The problem of finding optimal vector fields on a sphere is related to the problem of finding a minimizing cycle (vector field or not) in the homology class of the unit vector fields, considered as submanifolds of $U S^{n}$.

Our singular vector field arose out of the idea that volume minimizing cycles for the homology of a manifold should be found in the cut locus to a point of the manifold. 
For example, on $\mathbf{R} P^{n}$ the cut locus to a point, which is $\mathbf{R} P^{n-1}$, is volume minimizing in its homology class [1].

As another example, a theorem of Tasaki [9] states that, in a compact, simple, simply-connected Lie group, the cut locus of a point is volume-minimizing in its homology class. The cut loci of such Lie groups typically have one or more singular points, and are the exponential image of their tangent cones at one of the singular points.

Fix $\left(-p_{0}, v_{0}\right) \in U S^{n}$. (The reason for the apparently perverse choice of name, $\left(-p_{0}, v_{0}\right)$, will become clear when we define the generalized Pontryagin cycles later.) Define $W^{n}$ to be the set of all vectors obtained by parallel translating $v_{0}$ along any great circle of $S^{n}$ passing through $-p_{0}$. That is,

$$
W^{n}=\left\{\left(A\left(-p_{0}\right), A\left(v_{0}\right)\right) \mid A \in Q\right\},
$$

where $Q$ denotes the set of all transformations in $\mathrm{SO}(n+1)$ that simply rotate a 2-plane containing $p_{0}$. $W^{n}$ is a vector field on $S^{n}-\left\{p_{0}\right\}$. At $p_{0}$, the entire fiber $U_{p_{0}} S^{n}$ is included in $W^{n}$.

In the special case $n=2, W^{2}=\mathbf{R} P^{2}$ is the cut locus of $\left(-p_{0},-v_{0}\right)$ in $U S^{2}=\mathbf{R} P^{3}$.

We shall see in $\S 3$ that $W^{n}$ is a subvariety of $U S^{n}$ with one nonmanifold point, $\left(p_{0}, v_{0}\right)$. The preceding description of $W^{n}$ has the disadvantage that it gives the point $\left(-p_{0}, v_{0}\right)$ a distinguished role which it does not merit, and obscures the role of $\left(p_{0}, v_{0}\right)$. An equivalent definition of $W^{n}$ which gives $\left(p_{0}, v_{0}\right)$ a central role is

$$
W^{n}=\left\{\left(R_{x}\left(p_{0}\right), R_{x}\left(v_{0}\right)\right) \mid x \in S^{n}\right\},
$$

where $R_{x}$ denotes reflection of $\mathbf{R}^{n+1}$ across the hyperplane $x^{\perp}$. (The equivalence of the definitions follows from the fact that any rotation in a 2-plane containing $p_{0}$ may be written as $R_{x} R_{p_{0}}$ for some $x \in S^{n}$, and, conversely, the composition $R_{x} R_{p_{0}}$ is a rotation in the 2-plane spanned by $x$ and $p_{0}$. )

Proposition 3. The class $\left[W^{2 k+1}\right]$ generates $H_{2 k+1}\left(U S^{2 k+1}, Z\right)$, and the class [ $W^{2 k}$ ] generates $H_{2 k}\left(U S^{2 k}, Z / 2\right)$.

This follows from two simple facts: the maps

$$
\pi_{*}: H_{2 k+1}\left(U S^{2 k+1}, Z\right) \rightarrow H_{2 k+1}\left(S^{2 k+1}, Z\right),
$$

and

$$
\pi_{*}: H_{2 k}\left(U S^{2 k}, Z / 2\right) \rightarrow H_{2 k}\left(S^{2 k}, Z / 2\right)
$$

are isomorphisms; and the restriction $\pi: W^{n} \rightarrow S^{n}$ is a map of degree one. In particular, we have the following

Corollary 4. $W^{2 k+1}$ is in the same integral homology class as the unit vector fields on $S^{2 k+1}$.

In addition, we note that

Proposition 5. The cycle $W^{2 k+1}$ can be approximated by vector fields.

Using conformal stretching, we show how to construct vector fields that are in fact identical to $W^{2 k+1}$ outside a Riemannian $\varepsilon$-ball $U_{\varepsilon}$ on $S^{2 k+1}$ of radius $\varepsilon$ about $p_{0}$. Let $f: S^{2 k+1}-\left\{p_{0}\right\} \mapsto \mathbf{R}^{2 k+1}$ be stereographic projection. Let $V$ be a 
smooth unit vector field on $S^{2 k+1}$ with $V\left(-p_{0}\right)=v_{0}$. Let $V_{t}=\left(f^{-1}\right)_{*}\left(t f_{*}(V)\right)$. $\left(V_{t}\left(p_{0}\right)\right.$ is defined to be $V\left(p_{0}\right)$.) Then $V_{t}$ converges to $W^{2 k+1}$ as $t \mapsto \infty$. Away from $p_{0}, V_{t}$ comes to look more and more parallel along the great circles through $-p_{0}$. Thus, for large enough $t$, we can perturb $V_{t}$ and make it agree with $W^{2 k+1}$ outside $U_{\varepsilon}$.

\section{Facts about Pontryagin cycles}

3.1. Definition. Let $V_{k} \mathbf{R}^{n+1}$ denote the Stiefel manifold of ordered orthonormal $k$-frames in $\mathbf{R}^{n+1}$. Fix an orthonormal basis $\left\{e_{1}, e_{2}, \ldots, e_{n+1}\right\}$ in $\mathbf{R}^{n+1}$. Identify a $k$-frame $\left\{f_{1}, f_{2}, \ldots, f_{k}\right\}$ with the $(n+1)$-by- $k$ matrix having $f_{i}$ as its $i$ th column. Let $E=\left\{e_{1}, e_{2}, \ldots, e_{k}\right\}$. Metrize $V_{k} \mathbf{R}^{n+1}$ by declaring the projection $\pi: \mathrm{SO}(n+1) \rightarrow V_{k} \mathbf{R}^{n+1}$ given by $C \mapsto C E$ to be a Riemannian submersion.

Given a unit vector $x \in \mathbf{R}^{n+1}$, let $R_{x} \in \mathrm{O}(n+1)$ denote reflection across the hyperplane $x^{\perp}$. Fix a unit vector $z \in \operatorname{span}(E)^{\perp}$. Define the generalized Pontryagin cycle to be

$$
P_{k}^{n}=\left\{R_{x} R_{z} E \mid x \in S^{n}\right\} \subset V_{k} \mathbf{R}^{n+1} .
$$

(Notice that $R_{z} E=E$. We include $R_{z}$ in the notation to make it clear that $R_{x} E=R_{x} R_{z} E$ is in the orbit of $E$ under $\mathrm{SO}(n+1)$, and thus that $P_{k}^{n}$ actually lies in $V_{k} \mathbf{R}^{n+1}$ as defined.)

Proposition 6. $P_{k}^{n}$ is homeomorphic to $\mathbf{R} P^{n}$ with an $\mathbf{R} P^{n-k}$ smashed to a point and is smooth except at $E$.

To prove the homeomorphism, notice that $R_{x} R_{z} E=R_{x} R_{y} E$ if and only if either $x= \pm y$ or $x, y \in \operatorname{span}(E)^{\perp}$. So we get a degree 2 map $\mathbf{R} P^{n} \mapsto P_{k}^{n}$ with an equatorial $\mathbf{R} P^{n-k}$, the image of $\operatorname{span}(E)^{\perp}$, collapsing to a single image point, $E$. This also makes clear the smoothness assertion.

Notice that $P_{2}^{n}$ is the same as $W^{n}$, and that $P_{n}^{n}$ is one of the classical cycles in $\mathrm{SO}(n+1)$ defined by Pontryagin in [8].

\subsection{Group action. Define embeddings}

$$
\begin{aligned}
\kappa: \mathrm{SO}(k) & \rightarrow \mathrm{SO}(n+1), \\
A & \mapsto A_{\kappa}\left(\begin{array}{cc}
A & 0 \\
0 & I_{n+1-k}
\end{array}\right)
\end{aligned}
$$

and

$$
\begin{aligned}
\lambda: \mathrm{SO}(n+1-k) & \rightarrow \mathrm{SO}(n+1), \\
A & \mapsto A_{\lambda}=\left(\begin{array}{cc}
I_{k} & 0 \\
0 & A
\end{array}\right) .
\end{aligned}
$$

An action by isometries of $\mathrm{SO}(n+1) \times \mathrm{SO}(k)$ on $V_{k} \mathbf{R}^{n+1}$ is given by $(A, B)(C E)$ $=A C E B^{-1}=A C B_{\kappa}^{-1} E$. Note that $E B=B_{\kappa} E$ because, in matrix form, $E=\left(\begin{array}{c}I_{\kappa} \\ 0\end{array}\right)$. The isotropy subgroup of $E$ under this action is

$$
G=\left\{\left(A_{\lambda} B_{k}, B\right) \mid A \in \mathrm{SO}(n+1-k), B \in \mathrm{SO}(k)\right\} .
$$

$G$ is isomorphic to $\mathrm{SO}(n+1-k) \times \mathrm{SO}(k)$. The action of $G$ preserves $P_{k}^{n}$ because $\left(A_{\lambda} B_{\kappa}, B\right)\left(R_{x} R_{z} E\right)=R_{A_{\lambda} B_{\kappa}(x)} R_{z} E$. 
3.3. Tangent cone. Given $x_{0} \in \operatorname{span}(E)^{\perp}$ and $x_{1} \in \operatorname{span}(E)$, let $x(\theta)=$ $(\cos \theta) x_{0}+(\sin \theta) x_{1}$. The geodesic $R_{x(\theta)} R_{z}$ of $\mathrm{SO}(n+1)$ projects to a geodesic of $V_{k} \mathbf{R}^{n+1}$. Since every $x \in S^{n}$ may be written as $x(\theta)$ for some $x_{0}, x_{1}$, and $\theta$, we see that $P_{k}^{n}$ is a union of geodesics passing through the singular point $E$; that is,

Proposition 7. $P_{k}^{n}$ is the exponential image of $T C_{E} P_{k}^{n}$, its tangent cone at $E$.

A routine computation shows that

$$
\left.\frac{\partial}{\partial \theta}\left(R_{\cos \theta \mu(x)+\sin \theta \nu(y)} R_{z} E\right)\right|_{\theta=0}=-2\left(\begin{array}{c}
0 \\
x y^{t}
\end{array}\right),
$$

if $x$ and $y$ are unit vectors. To explain the notation: $\mu: \mathbf{R}^{n+1-k} \rightarrow \operatorname{span}(E)^{\perp}$ and $\nu: \mathbf{R}^{k} \rightarrow \operatorname{span}(E)$ are fixed isomorphisms. The tangent vectors in $T_{I} \mathrm{SO}(n+1)$ orthogonal to $\mathrm{SO}(n+k-1)$ may be written in the form $\left(\begin{array}{c}N-M^{t} \\ M\end{array}\right)$ where $N \in \mathfrak{s o}(k)$ and $M$ is an $(n+1)$-by- $k$ matrix. Then any tangent vector in $T_{E} V_{k} \mathbf{R}^{n+1}$ is of the form $\pi_{*}\left(\begin{array}{c}N-M^{t} \\ M\end{array}\right)=\left(\begin{array}{l}N \\ M\end{array}\right)$.

Consider the subspace

$$
V=\left\{\left(\begin{array}{c}
0 \\
M
\end{array}\right) \mid M \text { is an }(n+1) \text {-by- } k \text { matrix }\right\} \subset T_{E} V_{k} \mathbf{R}^{n+1} .
$$

The induced action of $G$ on $V$ is the same as the usual tensor product action of $\mathrm{SO}(n+1-k) \times \mathrm{SO}(k)$ on $\mathbf{R}^{(n+1-k) k}$. Clearly, $T C_{E} P_{k}^{n}$ is the cone over the orbit of a point under this action. Therefore,

Proposition 8. $T C_{E} P_{k}^{n}$ is the cone over $S^{n-k} \otimes S^{k-1}$.

Notice that we have also shown that $G$ acts transitively on the geodesics in $P_{k}^{n}$ through $E$.

We shall see in Proposition 12 that the tangent cone at the singularity of $P_{k}^{n}$ is volume minimizing for most $n$ and $k$. The exceptions are $P_{2}^{3}=W^{3}$, whose tangent cone is unstable; and $P_{2}^{4}=W^{4}$, for which stability of its tangent cone is undecided.

\subsection{Minimality.}

Proposition 9. The cycle $P_{k}^{n}$ is minimal at each smooth point.

We prove this by showing that the mean curvature of $P_{k}^{n}$ vanishes at each point, other than $E$, of the geodesic $g(\theta)=R_{(\cos \theta) z+(\sin \theta) e_{1}} R_{z} E$. Since $G(g-\{E\})=P_{k}^{n}-\{E\}, P_{k}^{n}-\{E\}$ is minimal.

Minimality along $g$ follows from a stronger statement: at each smooth point $x$ of $g$, there exists an orthonormal basis $\left\{z_{1}, \ldots, z_{k}\right\}$ for $T_{x} P_{k}^{n}$ such that $\beta\left(z_{i}, z_{i}\right)=0$ for $i=1, \ldots, k$. ( $\beta$ denotes the second fundamental form.) Then certainly the mean curvature of $P_{k}^{n}$, which is $\operatorname{tr} \beta$, vanishes.

The existence of the basis $\left\{z_{1}, \ldots, z_{k}\right\}$ again follows from a stronger fact: there exist $k$-dimensional and $(n+1-k)$-dimensional submanifolds of $P_{k}^{n}$ that intersect along $g$ and are totally geodesic inside $V_{k} \mathbf{R}^{n+1}$. In fact, the $k$-dimensional submanifold is $M=\left\{R_{x} R_{z} E \mid x \in \operatorname{span}(E, z)\right\}$ and the $(n+1-k)$-dimensional submanifold is

$$
\begin{aligned}
N & =\left\{R_{x} R_{z} E \mid x \in \operatorname{span}\left(e_{1}, e_{k+1}, e_{k+2}, \ldots, e_{n+1}\right)\right\} \\
& =\left\{\left\{f, e_{2}, e_{3}, \ldots, e_{k}\right\} \mid f \in \operatorname{span}\left(e_{1}, e_{k+1}, e_{k+2}, \ldots, e_{n+1}\right)\right\} .
\end{aligned}
$$


Notice that $P_{k}^{k}$ is totally geodesic in $S^{k+1}=V_{k} \mathbf{R}^{k+1}$ and the inclusion

$$
\varepsilon: V_{k} \mathbf{R}^{k+1} \hookrightarrow V_{k} \mathbf{R}^{n+1}
$$

given by identifying $\mathbf{R}^{k+1}$ with $\operatorname{span}(E, z)$ is totally geodesic. Thus $M$ is totally geodesic in $V_{k} \mathbf{R}^{n+1}$, since $M=\varepsilon\left(P_{k}^{k}\right)$. Also the inclusion

$$
\zeta: V_{1} \mathbf{R}^{n+2-k} \hookrightarrow V_{k} \mathbf{R}^{n+1}
$$

given by identifying $\mathbf{R}^{n+2-k}$ with $\operatorname{span}\left(e_{1}, e_{k+1}, e_{k+2}, \ldots, e_{n+1}\right)$ and augmenting a 1 -frame $\{f\}$ to a $k$-frame $\left\{f, e_{2}, e_{3}, \ldots, e_{k}\right\}$ is totally geodesic. Thus $N$ is totally geodesic in $V_{k} \mathbf{R}^{n+1}$, since $N=\zeta\left(V_{1} \mathbf{R}^{n+2-k}\right)$.

\section{Volume}

In this section we compute the volume of the singular unit vector field $W^{n}$ on $S^{n}$ and show that it is exceptionally small. We shall prove that

Theorem 10. The volume of $W^{n}$ is

$$
\operatorname{Vol} W^{n}=\frac{4^{n-1}}{\left(\begin{array}{c}
2 n-2 \\
n-1
\end{array}\right)} \operatorname{Vol} S^{n}=c(n-1) \operatorname{Vol} S^{n} .
$$

Recall that $c(k)=4^{k} /\left(\begin{array}{c}2 k \\ k\end{array}\right)$ is the lower bound for the normalized volume, Vol $V / \operatorname{Vol} S^{2 k+1}$, of any unit vector field $V$ on $S^{2 k+1}$.

The normalized volume of the Hopf vector field on $S^{2 k+1}$ is $2^{k}$. It follows immediately that

Corollary 11. For $k \geq 2$, Vol $W^{2 k+1}<\operatorname{Vol} H^{2 k+1}$.

Since, as noted in $\S 1, c(k) \sim \sqrt{\pi k}$, we find that $\operatorname{Vol} W^{2 k+1} / \operatorname{Vol} S^{2 k+1} \sim$ $\sqrt{2 \pi k}$. That is, the normalized volume of the singular unit vector field grows at the same rate as the lower bound.

To prove Theorem 10, we shall slice $W^{n}$ into round $(n-2)$-spheres and integrate the volume of these spheres. To name these spheres efficiently, we introduce some notation.

Given a point $p$ of a metric space $(M, d)$, let $\Sigma_{r}^{M}(p)$ denote the metric sphere of radius $r$ about $p$ :

$$
\Sigma_{r}^{M}(p)=\{q \in M \mid d(q, p)=r\} .
$$

Let $\pi: W^{n} \rightarrow S^{n}$ be the natural projection. If $A \subset S^{n}$, let $W(A)=\pi^{-1}(A)$. We shall use the description of $W^{n}$ as the set of all vectors obtained by parallel translating a fixed vector $v_{0} \in T_{p_{0}} S^{n}$ along any great circle passing through $p_{0}$.

Let $\gamma(t)=\exp _{p_{0}} t v_{0}$. This is a great circle in $S^{n}$.

Let $S^{n-1}(\phi)=\Sigma_{\phi}^{S^{n}}\left(p_{0}\right)$. For $\phi \in(0, \pi), W\left(S^{n-1}(\phi)\right)$ is diffeomorphic to $S^{n-1}$.

Let $S^{n-2}(\phi, \theta)=\Sigma_{\theta}^{S^{n-1}}(\gamma(\phi))$. For $\phi, \theta \in(0, \pi)$, the slice $W\left(S^{n-2}(\phi, \theta)\right)$ is isometric to a round sphere of radius $r=\sin \theta \sqrt{\sin ^{2} \theta+\cos ^{2} \theta(1-\cos \phi)^{2}}$.

To see that $W\left(S^{n-2}(\phi, \theta)\right)$ is round, note that the action of $\mathrm{SO}(n-1)$ on $\left(p_{0} \wedge v_{0}\right)^{\perp} \subset \mathbf{R}^{n+1}$ induces an action of $\mathrm{SO}(n-1)$ by isometries on $W^{n}$ that is 
transitive on the tangent planes to $W\left(S^{n-2}(\phi, \theta)\right)$. Therefore, $W\left(S^{n-2}(\phi, \theta)\right)$ has constant curvature.

To find the radius of $W\left(S^{n-2}(\phi, \theta)\right)$, let $g$ be a great circle of $W\left(S^{n-2}(\phi, \theta)\right)$, parametrized by length. Let $G(t)=W(g(t))=(g(t), v(t))$. Then the loop $G$ is traversed in time $T=2 \pi \sin \phi \sin \theta$. It is traversed at rate

$$
R=\left|G^{\prime}\right|=\sqrt{\left|\left(g^{\prime}\right)^{2}\right|+\left|\nabla_{g^{\prime}} v\right|}=\sqrt{1+\cos ^{2} \theta(1-\cos \theta / \sin \phi)^{2}}
$$

The length of $G$ is $R T$, and the radius of $W\left(S^{n-2}(\phi, \theta)\right)$ is $R T /(2 \pi)$.

Letting $d_{\phi} \sigma$ and $d_{\theta} \sigma$ denote arclength in the $\phi$ and $\theta$ directions, respectively, we have

$$
\operatorname{Vol} W^{n}=\int_{\phi=0}^{\pi} \int_{\theta=0}^{\pi} \operatorname{Vol} W\left(S^{n-2}(\phi, \theta)\right) d_{\phi} \sigma d_{\theta} \sigma .
$$

Substituting $d_{\phi} \sigma=d \phi, d_{\theta} \sigma=\sin (\phi / 2) d \theta$, and $\operatorname{Vol} S^{n-2}=\frac{n-1}{2 \pi} \operatorname{Vol} S^{n}$, we obtain

$$
\begin{aligned}
& \frac{\mathrm{Vol} W^{n}}{\operatorname{Vol} S^{n}}=\frac{n-1}{\pi} \int_{\phi=0}^{\pi} \int_{\theta=0}^{\pi / 2} \sin ^{n-2} \theta\left(\sin ^{2} \theta+\cos ^{2} \theta(1-\cos \phi)^{2}\right)^{(n-2) / 2} \\
& \times 2 \sin (\phi / 2) d \theta d \phi .
\end{aligned}
$$

Now substitute $u=(1-\cos \phi) / 2$ and $v=\sin ^{2} \theta$. Use binomial expansion and the beta function (see [5]) $B(x, y)=\int_{0}^{1} t^{x-1}(1-t)^{y-1} d t$ to get

$$
\begin{aligned}
\frac{\operatorname{Vol} W^{n}}{\operatorname{Vol} S^{n}} & =\frac{2^{n-2}(n-1)}{\pi} \sum_{j}\left(\begin{array}{c}
\frac{n-2}{j} \\
j
\end{array}\right)(-1)^{j} \\
& \times \int_{0}^{1} \int_{0}^{1} u^{n / 2-1+j} v^{n / 2-3 / 2+j}(1-u)^{-1 / 2}(1-v)^{-1 / 2} d u d v \\
= & \frac{2^{n-2}(n-1)}{\pi} \sum_{j}\left(\begin{array}{c}
\frac{n-2}{2} \\
j
\end{array}\right)(-1)^{j} B(n / 2+j, 1 / 2) B(n / 2-1 / 2+j, 1 / 2) \\
= & \frac{2^{n-2}(n-1)}{\pi} \sum_{j}\left(\begin{array}{c}
\frac{n-2}{2} \\
j
\end{array}\right)(-1)^{j} \frac{\pi}{n / 2-1 / 2+j} \\
= & 2^{n-2}(n-1) \int_{0}^{1} x^{(n-3) / 2}(1-x)^{(n-2) / 2} d x .
\end{aligned}
$$

Hence

$$
\frac{\operatorname{Vol} W^{n}}{\operatorname{Vol} S^{n}}=2^{n-2}(n-1) B\left(\frac{n-1}{2}, \frac{n}{2}\right) \text {. }
$$

The functions $g(k)=2^{k-1} k B(k / 2,(k+1) / 2)$ and $c(k)=4^{k} /\left(\begin{array}{c}2 k \\ k\end{array}\right)$ satisfy the same recursion relation,

$$
a_{k}=\frac{2 k}{2 k-1} a_{k-1}
$$

Also $g(1)=c(1)$. Therefore $g(k)=c(k)$ for all $k \geq 1$. Substituting $c(n-1)$ for $g(n-1)$ in the right-hand side of (1) gives us the desired formula and completes the proof of Theorem 10 . 


\section{Minimizing CONES} that

Recall that in $\S 3$ we found cycles $P_{k}^{n} \in V_{k} \mathbf{R}^{n+1}$. In this section we shall show

Proposition 12. The tangent cone at the singularity of $P_{k}^{n}$ is volume minimizing for $k \geq 3, n \geq 3$, and for $k=2, n \geq 5$.

Recalling that the tangent cone to $P_{k}^{n}$ is the cone over $S^{n-k} \otimes S^{k-1}$, this assertion follows as a corollary of the following theorem.

Theorem 13. The cones over $S^{q} \otimes S^{r}$ are volume minimizing for $q, r \geq 2$ and for $q=1, r \geq 3$.

$S^{q} \otimes S^{r}$ is defined to be the image of the map

$$
\begin{aligned}
S^{q} \times S^{r} & \rightarrow \mathbf{R}^{(q+1)(r+1)}, \\
(x, y) & \mapsto x \otimes y=x y^{t} .
\end{aligned}
$$

Thus the $i, j$ entry of $x y^{t}$ is $x_{i} y_{j}$. (We identify $\mathbf{R}^{(q+1)(r+1)}$ with the set of $(q+1)$-by- $(r+1)$ real matrices.) The cone over $S^{q} \otimes S^{r}$ is the set of all rank one $(q+1)$-by- $(r+1)$ matrices; $S^{q} \otimes S^{r}$ is the set of all rank one $(q+1)$-by- $(r+1)$ matrices of norm one. $S^{q} \otimes S^{r}$ is orientable precisely when $q$ and $r$ have the same parity. Some special cases: the cone over $S^{1} \otimes S^{1}$ is the cone over the Clifford torus inside $S^{3}$. The cone over $S^{1} \otimes S^{3}$ is the cone over $\mathrm{U}(2) \subset S^{7}$. Both of these cones have been previously classified-the former is unstable, and the latter was shown to be stable in [6].

We shall prove Theorem 13 as a corollary of a theorem of Lawlor [6]. Before we can state Lawlor's theorem, we must define some terms and notation.

Let $B^{k-1}$ be a compact minimal submanifold (not necessarily orientable) of $S^{n-1} \subset \mathbf{R}^{n}$, and denote by $C$ the cone over $B$ in $\mathbf{R}^{n}$ with vertex at the origin. Let $\nu B$ denote the normal bundle of $B \subset S^{n-1}$. If $p \in B$ and $v \in \nu_{p} B$ is a unit vector, define norm $(p, v)$, the normal radius of $B$ at $p$ in the direction $v$, to be the smallest positive value of $t$ such that $\exp _{p} t v \in B$, where $\exp$ is taken inside $S^{n-1}$. Define norm $(B)$, the minimum normal radius of $B$, to be $\min \left\{\operatorname{norm}(p, v) \mid p \in B, v \in \nu_{p} B\right\}$.

Lawlor's method of proving a cone minimizing is to construct a certain homothetically invariant volume-nonincreasing projection $\Pi: \mathbf{R}^{n} \rightarrow C$ that is the identity on $C$. If the construction can be carried out, then $\Pi$ vanishes outside a wedge of angular radius $\alpha$ around $C$. The necessary vanishing angle $\alpha$ is the solution to a differential equation involving the norm of the second fundamental form of $B$ and the dimension of $C$. This equation constitutes a local condition for the construction to work. If the equation is unsolvable, then under certain conditions $C$ can be shown to be unstable. However, the equation may be unsolvable and yet $C$ might still be stable, or even minimizing. Even if the equation is solvable, there may be global obstructions to constructing the projection. However, the following is true:

Theorem 14 (Lawlor). If the minimum normal radius of $B$ is greater than or equal to twice the vanishing angle of $B$, then $C$ is volume minimizing both in the class of integral currents and in the class of integral currents modulo 2.

We shall apply Lawlor's theorem to the case $B=S^{q} \otimes S^{r}, k=q r+1$, $n=(q+1)(r+1)$. 
Since $S^{q} \otimes S^{r}$ is homogeneous, we may compute its minimum normal radius by computing the normal radius at a single point. Fix orthonormal bases $\left\{e_{0}, e_{1}, \ldots, e_{q}\right\}$ and $\left\{f_{0}, f_{1}, \ldots, f_{r}\right\}$ for $\mathbf{R}^{q+1}$ and $\mathbf{R}^{r+1}$, respectively. Let $p=e_{0} \otimes f_{0}$. We may identify $e_{i} \otimes f_{j}$ with tangent vectors to $\mathbf{R}^{(q+1)(r+1)}$. In particular, $\left\{x_{i}=e_{i} \otimes f_{0}\right\}_{i \geq 1} \cup\left\{y_{j}=e_{0} \otimes f_{j}\right\}_{j \geq 1}$ is an orthonormal basis for $T_{p}\left(S^{q} \otimes S^{r}\right)$, and $\left\{z_{i j}=e_{i} \otimes f_{j}\right\}_{i, j \geq 1}$ is an orthonormal basis for $\nu_{p}\left(S^{q} \otimes S^{r}\right)$.

For every nonzero $v \in \nu_{p}\left(S^{q} \otimes S^{r}\right)$ and every nonzero $t$, the matrix $p+t v$ is of rank two, hence $\operatorname{norm}(p, v) \geq \pi / 2$. (Notice that, for $\pi / 2<t<\pi / 2$, $\exp _{p} t v$ is the projection onto $S^{n-1}$ of $p+(\tan t) v$.) Since $\exp _{p}(\pi / 2)\left(e_{0} \otimes f_{1}\right)=$ $e_{0} \otimes f_{1}$, we see that $\operatorname{norm}(p, v)=\pi / 2$. Hence the minimum normal radius of $S^{q} \otimes S^{r}$ is $\pi / 2$.

We use a table computed by Lawlor [6] to estimate the vanishing angle, $\alpha$, of $S^{q} \otimes S^{r}$. To use this table, we need to know two numbers: the dimension, $k$, of $C$, and the minimum over all points $p \in B$ and unit vectors $v \in \nu_{p} B$ of $\left\|\beta^{v}\right\|$. (Here, $\beta^{v}$ denotes the second fundamental form of $B$ in the direction $v$.) Again, since $S^{q} \otimes S^{r}$ is homogeneous, we need only compute $\left\|\beta^{v}\right\|$ at $p=e_{0} \otimes f_{0}$. A routine calculation shows that

$$
\beta\left(x_{i}, x_{j}\right)=0, \quad \beta\left(y_{i}, y_{j}\right)=0, \quad \beta\left(x_{i}, y_{j}\right)=z_{i j} .
$$

If $v=\sum a_{i j} z_{i j}$ is a unit vector, then $\beta^{v}=\langle\beta, v\rangle$ and $\left\|\beta^{v}\right\|^{2}=\sum a_{i j}+\sum a_{i j}=$ 2 , independent of $v$ !

For $k \geq 5$ and $\min \left\|\beta^{v}\right\|^{2}=2$, Lawlor's table shows that the vanishing angle is less than or equal to $27.3^{\circ}$. (For fixed $\min \left\|\beta^{v}\right\|^{2}$, the vanishing angle decreases as $k$ increases.) Therefore, when $q r+1 \geq 5$, the minimum normal radius, $90^{\circ}$, is at least twice the vanishing angle and the cone over $S^{q} \otimes S^{r}$ is minimizing. This happens when $q, r \geq 2$ and when $q=1, r \geq 3$.

When $k \leq 4$ and $\min \left\|\beta^{v}\right\|^{2}=2$, the vanishing angle does not exist and therefore the version of Lawlor's theorem given here does not apply. While the Clifford torus is known to be unstable, there remains the following

Question 15. Is the cone over $S^{1} \otimes S^{2}$ stable or unstable? If it is stable, is it volume minimizing?

\section{ACKNOWLEDGMENT}

The results in this paper were proved in the author's Ph.D. thesis [7] at the University of Pennsylvania, and the author expresses her deep gratitude to Herman Gluck for many valuable discussions. Thanks are also due the referee for helpful comments regarding the proof of Proposition 5. Revisions of this article were carried out in part while the author was supported by the North Atlantic Treaty Organization under a Grant awarded in 1988.

\section{REFERENCES}

1. A. T. Fomenko, Minimal compacta in Riemannian manifolds and the Reifenberg conjecture, Math. USSR-Izv. 6 (1972), 1037-1066.

2. H. Gluck, F. Warner, and W. Ziller, The geometry of the Hopf fibrations, Enseign. Math. 32 (1986), 173-198.

3. Herman Gluck and Wolfgang Ziller, On the volume of a unit vector field on the three sphere, Comment. Math. Helv. 61 (1986), 177-192. 
4. David L. Johnson, Volumes of flows, Proc. Amer. Math. Soc. 104 (1988), 923-932.

5. Donald E. Knuth, The art of computer programming, vol. 1: Fundamental algorithms, 2nd ed., Addison-Wesley, Reading, Mass., 1973.

6. Gary Lawlor, A sufficient criterion for a cone to be area minimizing, Ph.D. thesis, Stanford Univ., 1988.

7. Sharon L. Pedersen, Optimal vector fields on spheres, Ph.D. thesis, Univ. of Pennsylvania, 1988.

8. L. Pontrjagin, Homologies in compact Lie groups, Mat. Sb. 48 (1939), 389-422.

9. Hiroyuki Tasaki, Certain minimal or homologically volume minimizing submanifolds in compact symmetric spaces, Tsukuba J. Math. 9 (1985), 117-131.

Department of Mathematics, Bowdoin College, Brunswick, Maine 04011

E-mail address: pedersen@polar.bowdoin.edu 